\title{
Phased Arrays in Satcom
}

... why?

- Flexibility!!

$\rightarrow$ power allocation per beams much more easy than in SFPB

$\rightarrow$ coverage modification in active Phased Arrays

- Robustness

$\rightarrow$ Graceful degradation (you can loss elements with minimum impact on the radiation diagram)

how?

\section{- Digital Beamforming}

$\rightarrow$ Trend of today's DRA (Quantum from EUTELSAT, for example)

$\rightarrow$ The maximum flexibility $\rightarrow$ but at the expenses of large power consumption!

$\rightarrow$ for limited number of beams

- Analog Beamforming ..may be photonics?

$\rightarrow$ A number of options implemented .... All of them bulky 


\section{Phased Arrays in HTS ... what is needed?}

- Hundreds of beams \& antenna elements (HTS) !!!!

- High accuracy in phase and amplitude weights

$\rightarrow$ Side lobes, beam pointing...

- Extremally complex HW

$\rightarrow$ Antenna, power amplifiers, BFN, distribution together!!

$\rightarrow$ Calibration is a challenge

- Broadband (BW $>500 \mathrm{MHz}$, probably $>2 \mathrm{GHz}$ with beam-hopping) $\rightarrow$ beamsquint could be an issue.

- Linearity requirements

$\rightarrow$ all antenna elements radiates all the beam signals

- Losses \& NF

$\rightarrow$ in Tx, losses to be compensated in the antenna (Power, complexity, reliability)

$\rightarrow$ in Rx, NF impacts on the G/T... so you need more gain! 


\section{How photonics can "try" to help?}

- Hundreds of beams \& antenna elements (HTS) !!!!

$\rightarrow$ scalable structure... probably fixed BFN could be enough

- High accuracy in phase and amplitude weights

$\rightarrow$ optical heterodyne probably not enough... but homodyne yes?

- Extremely complex HW

$\rightarrow$ use optical fiber to remove the BFN from the antenna assembly

$\rightarrow$ try to maintain control complexity as simple as possible

- Broadband (BW > $500 \mathrm{MHz}$, probably $>2 \mathrm{GHz}$ with beam-hopping)

$\rightarrow$ TTD (full or partial) could help... but only needed for very large BWs

- Linearity requirements

$\rightarrow$ linearity of the photodiodes should be low!

- Losses \& NF

$\rightarrow$ probably is the major issue... (remember we are thinking in hundreds of antennas and beams)

$\rightarrow$ we can try to increase power... but with certain limit (especially in PIC), and at the expenses of power consumption. 


\section{Photonic RF beam-forming. FIXED COVERAGE From ICSO 2014}

\section{One dimension Butler Matrix (8X8)}

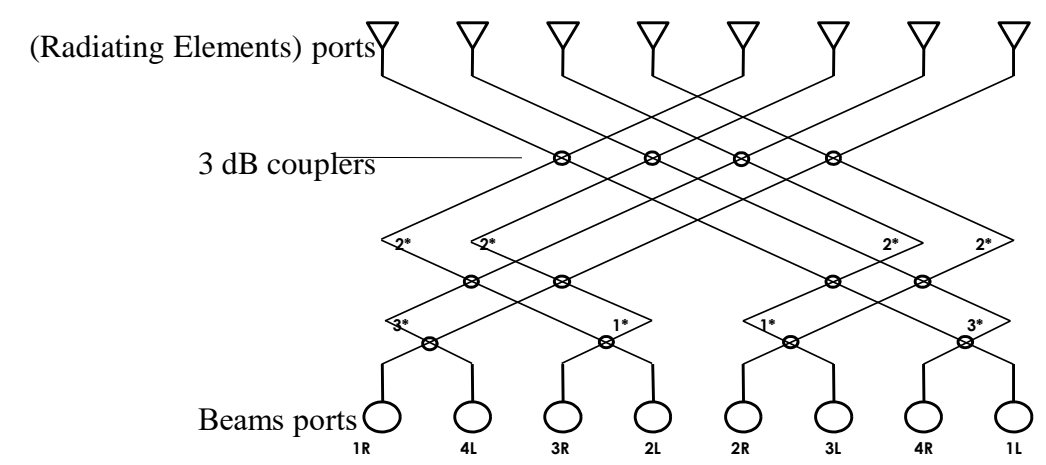

For 8 regularly spaced beams \& 8 RE (Radiating Elements), it reduces NB of couplers from 112 in $2 \mathrm{D}$ structure to 12 in planar structure !

\section{Two dimensions Butler Matrices set}

\section{n'a}

$$
\text { Layer -2 }
$$

Layer - 1

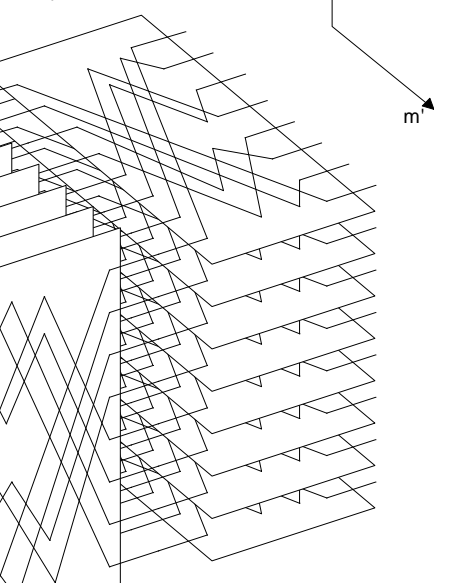

For 64 regularly spaced beams (in $2 \mathrm{D}=8^{\star} 8$ ) \& 64 $\mathrm{RE}$, it needs only 192 couplers, instead of 8064 for classical beam-former with 1 slide per beam \& $\mathrm{RE}$, or 1792 if split in 2 sets as Butler!

\section{Antenna Patterns}

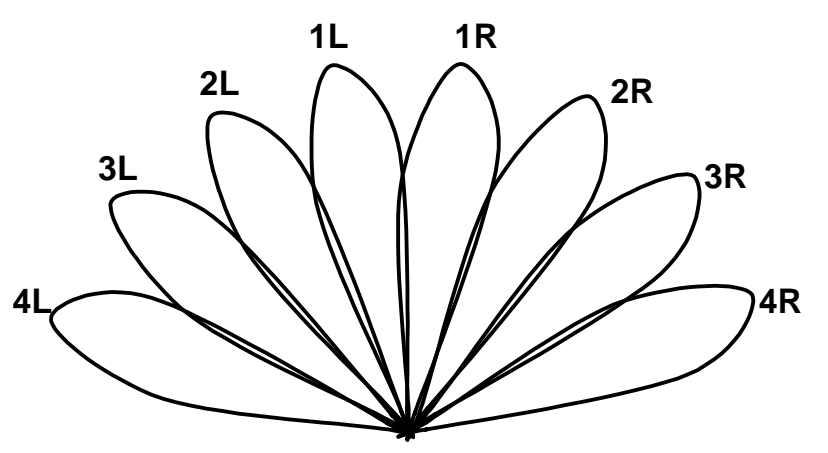

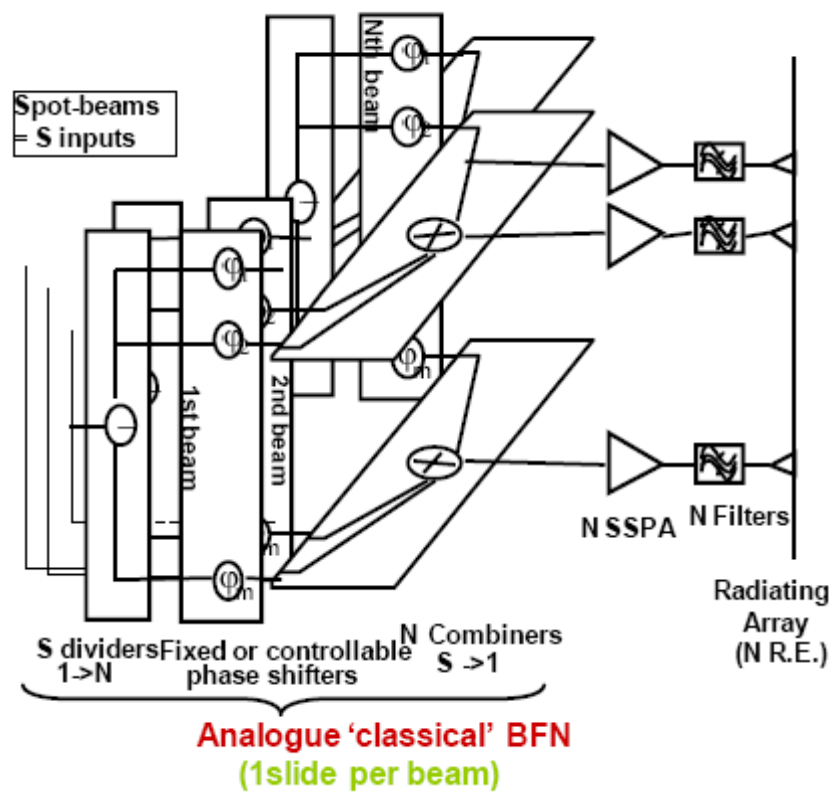




\section{Photonic RF beam-forming. FIXED COVERAGE From ICSO 2014}
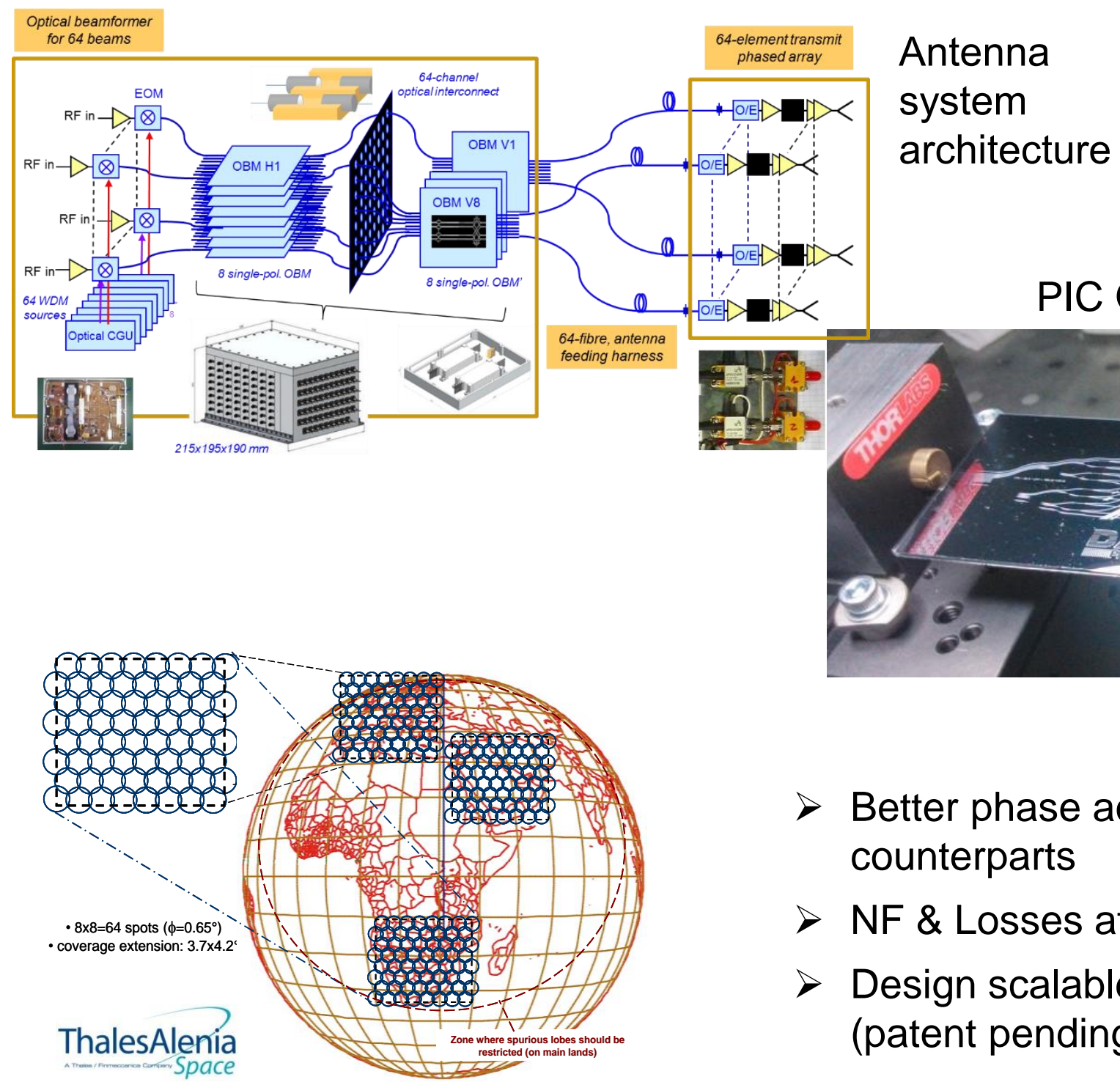

PIC Optical Butler Matrix 8x8
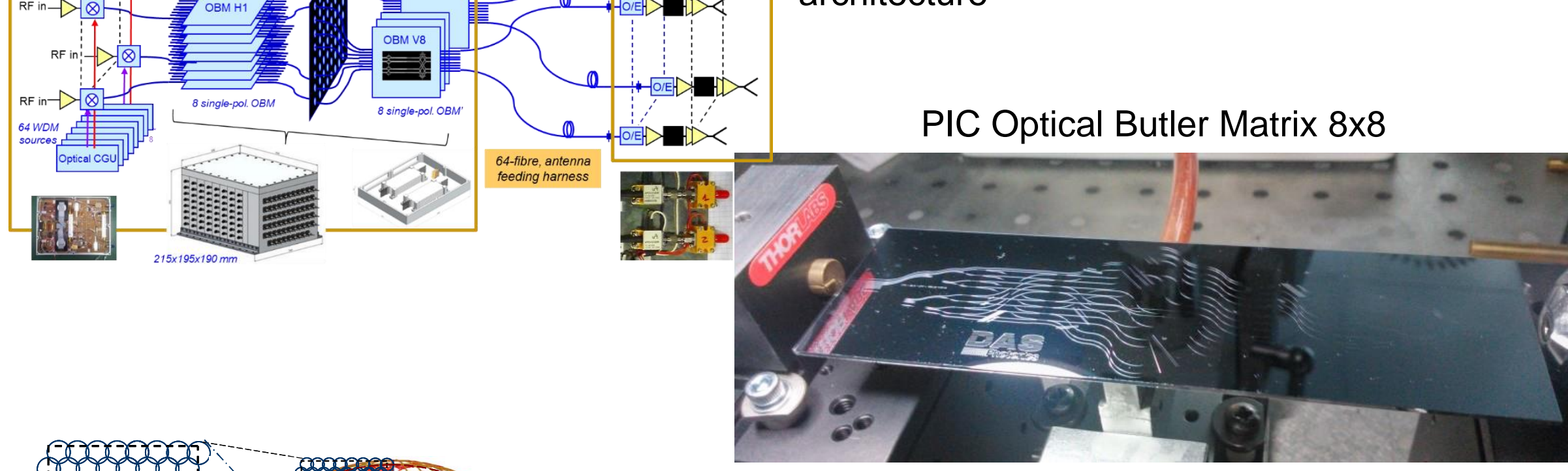


\section{Photonic RF beam-forming. Tunable TTD}

PIC \& Fiber Optics distribution RADARSAR applications,

- optical signal distribution to the antenna,

- the true-time-delay control of the signal for each antenna element (PICS): TX/RX

- optical harness for deployable antennas

- antenna array module in $X$ band.

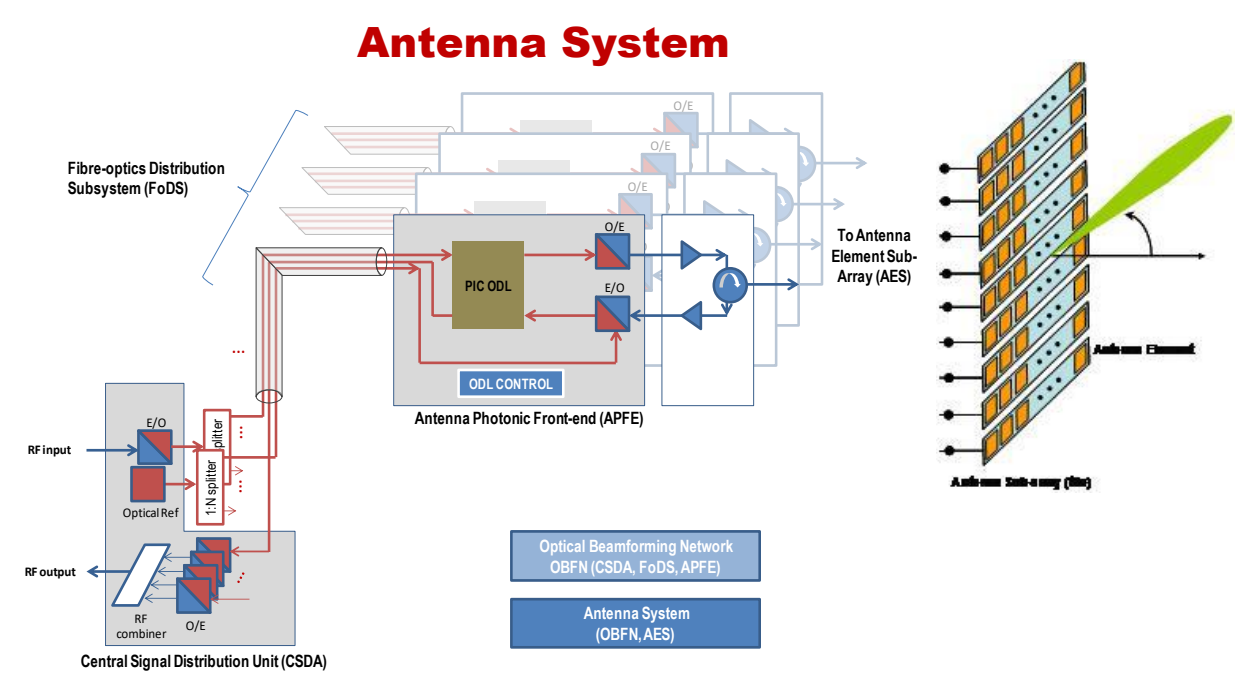

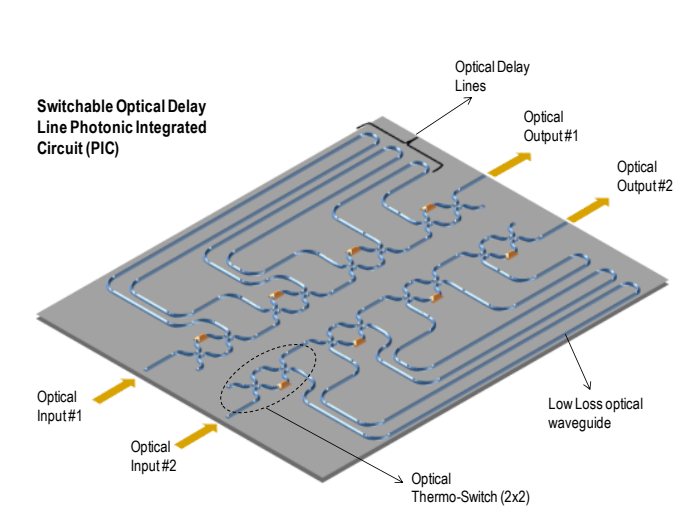

FP7 GAIA Project
PHOTONIC CHIP

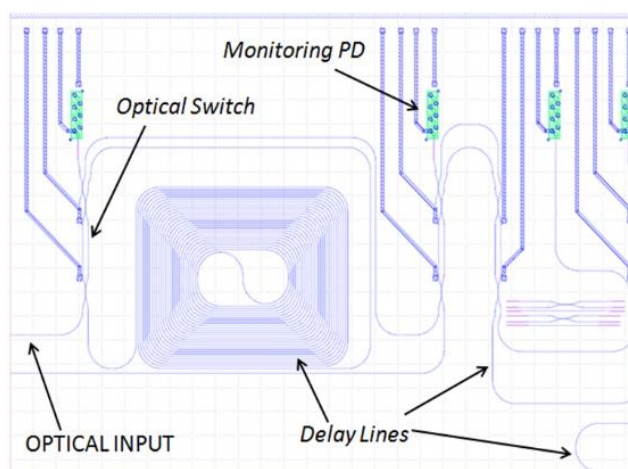

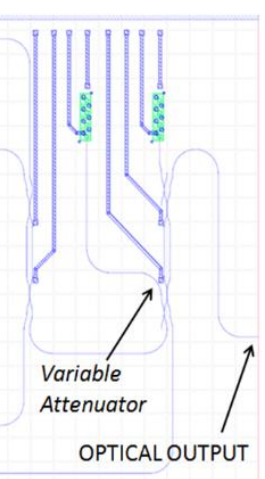

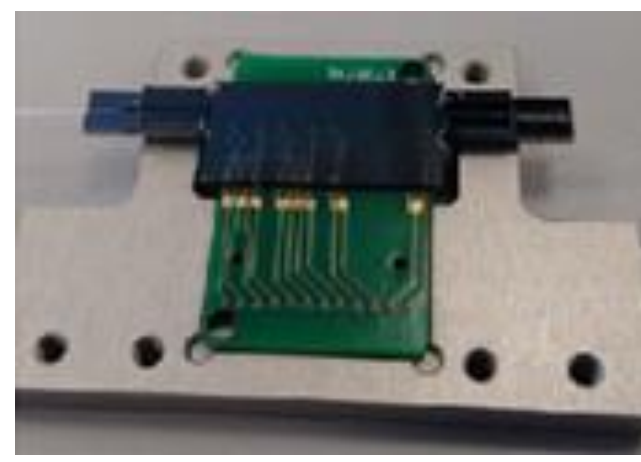

